\title{
Evolution and molecular epidemiology of foot-and-mouth disease virus in China
}

\author{
BAI XingWen *, LI PingHua, BAO HuiFang ${ }^{*}$, LIU ZaiXin", LI Dong, LU ZengJun, CAO YiMei, \\ SHANG YouJun, SHAO JunJun, CHANG HuiYun, LUO JianXun \& LIU XiangTao
}

\begin{abstract}
State Key Laboratory of Veterinary Etiological Biology, National Foot-and-Mouth Disease Reference Laboratory, Engineering Research Center of Biological Detection of Gansu Province, Lanzhou Veterinary Research Institute, Chinese Academy of Agricultural Sciences, Lanzhou 730046,
\end{abstract} China

Received April 7, 2011; accepted May 12, 2011

\begin{abstract}
Foot-and-mouth disease (FMD), caused by foot-and-mouth disease virus (FMDV), is considered one of the most important viral diseases of cloven-hoofed animals, causing severe economic losses in affected regions of the world. Three serotypes (A, O, and Asia 1) of FMDV have been identified in China since 1958. In addition, the occurrence of novel subtypes within these serotypes has made the epidemiology of FMDV more complicated over the last few years. In this review, we summarize the history and the current epidemiological situation in China, genetic diversity (e.g., quasispecies dynamics, antigenic heterogeneity, and functional constraints), intertypic recombination, and the evidence for positive selection of different FMDV serotypes. We also assess these genetic data to understand the origin, evolution, and transmission of FMDV, the findings of which may be useful in developing control measures for future epidemics.
\end{abstract}

foot-and-mouth disease virus, phylogenetic analysis, recombination, positive selection

Citation: $\quad$ Bai X W, Li P H, Bao H F, et al. Evolution and molecular epidemiology of foot-and-mouth disease virus in China. Chinese Sci Bull, 2011, 56: 2191-2201, doi: 10.1007/s11434-011-4563-3

Foot-and-mouth disease (FMD) is an acute, highly contagious disease of cattle, pigs, sheep, goats, and many cloven-hoofed wild animals [1]. Despite low mortality rates, FMD causes significant economic losses because of the negative effects livestock production and international trade restrictions on animals and animal products [2]. Foot-andmouth disease virus (FMDV), the etiological agent, is a positive-strand RNA virus that belongs to the genus Aphthovirus of the family Picornaviridae. There are 7 distinct serotypes of FMDV (A, O, C, Asia 1, and South African territories [SAT] 1-3) and multiple subtypes reflecting significant genetic variability in each serotype [3-5]. Despite intensive research efforts, FMDV still remains enzootic in many regions of the world. It is endemic and extensive in some parts of Asia, Africa, and sporadic in certain member states of the European Union [6-10].

*Corresponding authors (email: baixingwen@163.com; baohf1105@163.com; liuzaixin @ hotmail.com)
In China, 3 of the 7 serotypes of FMDV (A, O, and Asia 1) have been isolated and characterized. Here, we retrieved all the nucleotide sequences of FMDVs isolated from mainland China, Hong Kong, and Taiwan (no data available for Macau) from the NCBI/GenBank database to understand the evolution and molecular epidemiology of FMDV. These genetic data were collected with the aim of (i) reviewing the history of FMD outbreaks; (ii) describing the current situation of FMD epidemics and their effective control; (iii) determining the genomic regions corresponding to important biological functions; and (iv) conducting phylogenetic analysis to detect potential recombination and selection events.

\section{The history of FMD outbreaks in China (1958-1996)}

The first reports of FMDV isolates were in 1958. A 
serotype $\mathrm{O}$ isolate $(\mathrm{O} / \mathrm{Akesu} / \mathrm{CHA} / 58)$ was collected from Akesu prefecture [11], a serotype A isolate $(\mathrm{A} / \mathrm{XJ} / \mathrm{KT} /$ CHA/58) was collected from Aketao county of the Xinjiang Uygur Autonomous Region [12], and a serotype Asia 1 isolate (Asia 1/YN/BS/CHA/58) was collected from Baoshan in Yunnan Province [13]. Since then, only a few minor FMD outbreaks (of serotype A) were detected in Gansu Province in 1962 and in the Inner Mongolia Autonomous Region in 1960 and 1964 (involving 2 infected premises (IPs)). The social, economic, and political problems in mainland China reduced the effectiveness of veterinary services up until the era of reform and opening-up. Around 20 years later, strain $\mathrm{O} / \mathrm{GD} / \mathrm{China} / 86$ strain was reportedly isolated from swine $[14,15]$. Hong Kong has reported the presence of serotypes O and Asia 1 from 1970-1996 and 1974-1980, respectively.

\section{Current situation regarding FMD epidemics in Taiwan, Hong Kong, and mainland China (1997-2010)}

\subsection{Serotype O}

Serotype $O$ is the most prevalent and widely distributed serotype of FMDV globally, especially throughout the Middle East-South Asia (ME-SA) region [16]. Japan, Korea, Mongolia, Russia, the United Kingdom, France, and South Africa reported the presence of serotype O FMDV from 2000-2006, with all of these isolates belonging to the PanAsia group of strains [7,17-21]. According to the information provided by the Food and Agriculture Organization (FAO), the reoccurrence of FMDV serotype $\mathrm{O}$ in Japan, Korea, and Mongolia was confirmed in April 2010 (http://www.wrlfmd.org).

In 1997, an epizootic in Taiwan, a province of China, was caused by a serotype O FMDV. Disease was restricted to host pigs (family Suidea), and more than 4 million pigs had to be slaughtered [22-24]. FMD was endemic in Hong Kong between 1999 and 2004 [25-28]. In our survey, the presence of serotype $\mathrm{O}$ virus was reported in mainland China during a similar period (1999-2005) [29-33]. Epidemiological data and genetic characterization of circulating field viruses, as well as their relationships to vaccine strains that are employed in these areas, have been clarified. Efforts to control the movement of virus have been implemented by systematic vaccination. However, outbreaks of serotype O reoccurred in Taiwan in 2009 and in Hong Kong in May 2010 [34]. FMD outbreaks in mainland China have been prevalent. On 22 February 2010, the first confirmation of the disease was declared in Baiyun district in Guangdong Province. Up until late 2010, more than 18 IPs in 9 provinces had been detected (http://web.oie.int/wahis/public.php).

\subsection{Serotype Asia 1}

Members of this serotype are primarily restricted to Asia with recent incursions in the ME region, Korea (2007), Mongolia (2005), Russia (2005), Turkey, and Greece (2000) [35-38].

In 2005, FMDV of serotype Asia 1 was detected in cattle in Wuxi in Jiangsu Province. The disease spread rapidly and widely throughout mainland China, and a total of 46 IPs in 17 provinces reported outbreaks of FMDV serotype Asia 1 from 2005-2009 [39-42]. The presence of serotype Asia 1 virus was also reported in Hong Kong in March 2005 [43]. These outbreaks were gradually controlled.

\subsection{Serotype A}

Of the 7 serotypes, serotype A displays the highest antigenic diversity, and recombination in this serotype occurs more frequently than in other serotypes. However, the origin of the recombinant virus remains unclear $[44,45]$. Epidemiological investigations have revealed new outbreaks of FMDV serotype A in dairy cattle and deer in Korea since January 2010. These cases were confirmed by the isolation and identification of samples, clinical signs, positive results for antigen detection by an enzyme-linked immunosorbent assay, and nucleotide sequencing of the complete capsid protein VP1 coding region.

Since 1958, there have been no major outbreaks reported of this serotype in China. However, there is still no explanation for the emergence of an epidemic of FMDV serotype A in Hubei and Shanghai in early 2009. Phylogenetic analysis revealed that these isolates belonged to the South-East Asia (SEA) topotype. Subsequent outbreaks of FMD have spread to $10 \mathrm{IPs}$ in 8 provinces (http://web.oie.int/wahis/public.php).

\section{Molecular genetic characterization of FMDV VP1 protein, 3A protein, and pseudoknots}

\subsection{VP1}

VP1 is the preferred region for comparison of FMD outbreak strains because of its significance for the analyses of serotype specificity, the dynamics of quasispecies evolution, the occurrence of antigenic heterogeneity, and the interactions of FMDV with cells $[4,46,47]$. In comparison with the VP1 sequence of serotype $O$, a deletion exists at nucleotide positions 427-432 of the VP1 sequence of serotype Asia 1 and a deletion/insertion exists at nucleotide positions 430-432/586-588 of the VP1 sequence of serotype A. Amino acid residues 133-158 (inclusive of VP1) form a G-H loop structure constituting important antigenic determinants, which protrude from the viral surface [48]. The tripeptide RGD motif within the G-H loop is a cellular recognition site which combines with integrins during the process of virus-receptor interactions. While sequences surrounding the RGD motif within the G-H loop vary, the RGD motif itself is highly conserved [49,50]. Storey et al. 
[51] found that a second RGD motif in the VP1 capsid protein of SAT 1-type FMDV field isolates is not essential for attachment to susceptible cells. The coevolution of antigenicity and host cell tropism of FMDV suggests that the antigenic changes could be associated with alterations in cell receptor usage [52]. Glycosaminoglycan heparin sulfate (HS) has been reported as another receptor for a subtype of FMDV serotype O [53]. The rapid evolution of these antigenic sites in FMDV in nature hinders synthetic vaccines from providing efficient protection [54-56]. Phenotypic variability of viral RNA is strongly influenced by high mutation rates and quasispecies dynamics [57].

For comparison of the full and partial VP1 nucleotide sequences of viral isolates, a phylogenetic tree was constructed using the neighbor-joining algorithm [58]. The data revealed 113 isolates of the FMDV serotype $\mathrm{O}$ from China (7 isolates with partial VP1 sequences), grouping in the Cathay, PanAsia, and SEA topotypes. The porcinophilic lineage strains (of the Cathay topotype, including the HKN70, HKN90, and TAW97 genotypes) of FMDV were the pathogens responsible for the pandemics in Hong Kong and Taiwan during the 1990s, and the recent outbreaks in the mainland and Hong Kong (2010) were caused by the SEA topotype (Mya98 strain) of FMDV serotype O [59]. A total of 35 isolates of FMDV serotype Asia 1 from China (two isolates with partial VP1 sequences) were subdivided into the CHA58, Group II, HKN74 and Group IV (SEA topotype), and Group V genotypes (Figure 1). Amino acid sequence alignments have shown that the RGD motif is lacking in some virus isolates (the tripeptide SGD motif, $\mathrm{O} / \mathrm{Akesu} / \mathrm{CHA} / 58$ ) and certain variants use an RGD-independent infection mechanism that does not require binding to the surface HS. This suggests the potential use of at least 3 alternative receptors for entry of FMDV into target cells [60]. FMDV mutability and the simultaneous existence of several motifs (RGD, RDD, and RSD) within FMDV populations in vitro may contribute to memory in viral quasispecies [41] (partly unpublished data).

Phylogenetic statistical analysis clearly showed that groups of viruses share common geographical characteristics and epidemic histories. However, these data do not provide information regarding the origin of viruses and the sources of occasional global pandemics. The phylogenetic tree revealed that certain isolates may play a significant role in the genetic evolutionary dynamics of FMDV. Isolate Asia 1/YN/ML/CHA/2000 (CHA58 genotype) collected from unvaccinated cattle, indicates that epidemiological investigation is responsible for not only new outbreaks caused by novel FMDV strains, but also the re-emergence of new cases caused by "old" virus isolates.

\section{$3.23 \mathrm{~A}$}

$3 \mathrm{~A}$ protein is likely to play a role in viral virulence and the determination of host range. Changes in protein $3 \mathrm{~A}$ have been reported in several virus isolates belonging to serotypes $\mathrm{O}$ and $\mathrm{C}$, which have become attenuated in cattle but remain pathogenic in pigs. Molecular characterization of these viruses revealed that amino acid deletions (10-, 11-, 19-, or 20-codons) and series of substitutions occurred in the $\mathrm{C}$-terminal half of the $3 \mathrm{~A}$ coding region $[17,23,61]$. Deletions and mutations in protein $3 \mathrm{~A}$ appear to be responsible for this particular phenotype, and they affect RNA synthesis in keratinocytes of swine origin less than those of bovine origin [17].

In a comparison of the $3 \mathrm{~A}$ nucleotide sequences of 80 FMDV isolates (including 47 isolates collected in China), an unrooted neighbor-joining tree showed coevolution in accordance with the sequence alignment based on the nucleotides in the VP1 region (Figure 2). There were 10 amino acid deletions of codons at positions 93-102 in the porcinophilic lineage (Figure 2). In addition, isolate Asia 1/HKN/CHA/2005 was found to have the highest level of similarity with PanAsia strains in the $3 \mathrm{~A}$ region $(95.1 \%$ on average), indicating a closer relationship between Asia 1/HKN/CHA/2005 and PanAsia strains. The series of amino acid substitutions originated in the porcinophilic lineage and resulted in fitness gain or loss (from quantitative variation to qualitative alteration), which may affect cell tropism and host range during FMDV evolution. In some cases, the Q44R mutation in the $3 \mathrm{~A}$ protein was associated with the adaptation of FMDV to guinea pigs [62].

Fifteen amino acid deletions (131-145) of OMIII (artificially attenuated from strain O/Akesu/CHA/58) were displayed in the $3 \mathrm{~A}$ protein (Figure 2). Another strain of serotype $\mathrm{O}$ isolated from bovine and identified during the 35 th passage in swine cells, contained 18 amino acid deletions (85-102) in the $3 \mathrm{~A}$ region (unpublished data). Baranowski et al. $[60,63,64]$ documented that the FMDV clone C-S8c1, a virus recovered after 100 persistent passages of carrier BHK-21 cells, resulted in increased virulence of the BHK-21 cells and altered cell tropism. Several amino acid substitutions were identified in this clone around the G-H loop. These data showed that large population passages of FMDV may lead to host cell tropism alterations [4]. Interestingly, Oem et al. [65] also showed that a Korean 2002 isolate (PanAsia strain, O/AS/SKR/2002), which contained an intact $3 \mathrm{~A}$ coding region, caused an infection that was highly virulent and contagious in pigs, but limited in cattle. Analysis of this virus found that mutations in other regions correlated with changes in the VP1 and $3 \mathrm{~A}$ coding regions of the FMDV genome, and may indicate alterations in the host range.

Furthermore, $3 \mathrm{~A}$ is a transmembrane protein that contains hydrophobic sequences (61-76 aa) and the expression of FMDV 3A protein caused a disruption of the Golgi apparatus [66]. Structural prediction of the $3 \mathrm{~A}$ protein revealed a conserved N-terminal helix (1-92 aa) and a C-terminal random-coil conformation (93-153 aa) similar to 


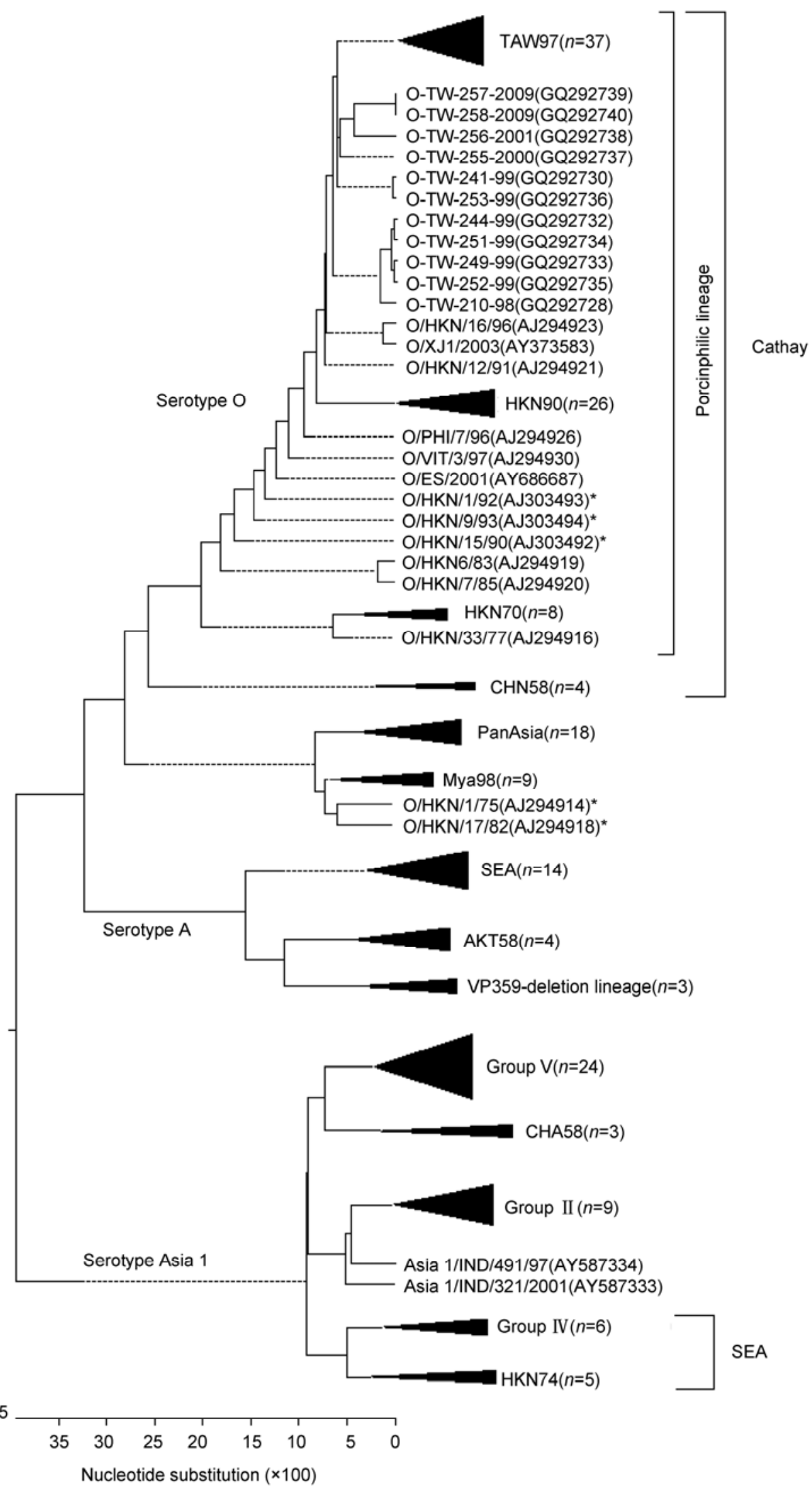

Figure 1 Phylogenetic tree of the VP1 nucleotide sequences of FMDV generated using the MegAlign software. The 197 FMDV isolates comprised 127, 49, and 21 isolates of serotype O, Asia 1 and A, respectively. * The partial VP1 sequence data of these isolates. $n$ Number of isolates in each group. 

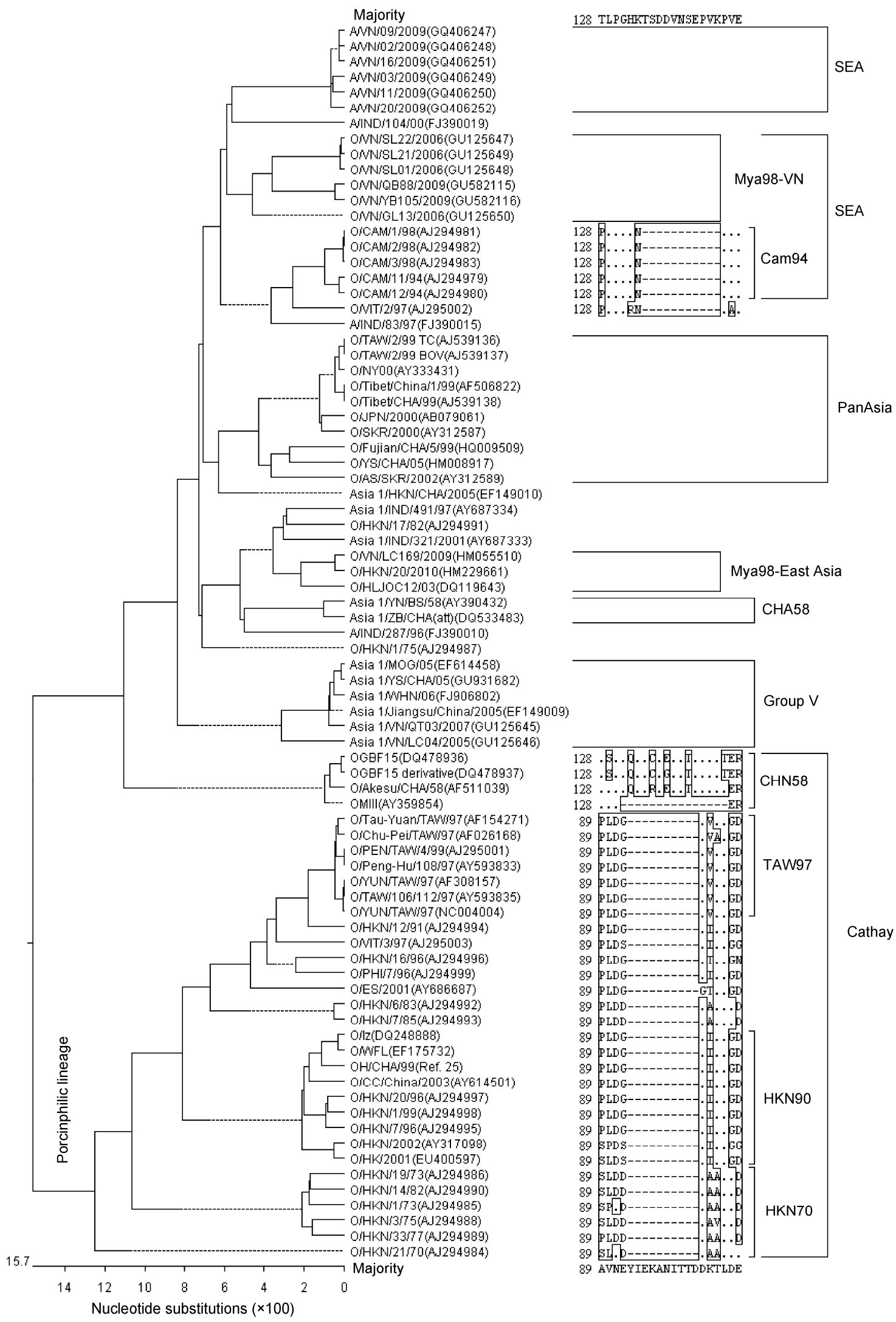

Figure 2 Unrooted neighbor-joining tree generated from the nucleotide sequences of the $3 \mathrm{~A}$ region and alignment of the amino acid sequences of the $3 \mathrm{~A}$ protein of FMDV isolates (from codon positions 89 to 108, and 128 to 147, respectively). 
phosphoprotein enriched in astrocytes of $15 \mathrm{kD}$ (PEA-15), indicating that FMDV $3 \mathrm{~A}$ is an "on-off switch" required for manipulating integrin activation in a Ras-dependent manner (Zhang et al., unpublished data).

\subsection{Pseudoknots (PKs)}

PKs have been predicted to be involved in conserved structures that are highly tolerant to changes at the 3 termini of the poly $(\mathrm{C})$ tract of FMDV genomes. PKs are believed to play a crucial role in the RNA replication cycle and the origin of polyprotein synthesis $[67,68]$. There is some evidence of nucleotide sequence deletions in this region in serotypes
A, C, and O [69-71]. These data raise interesting possibilities regarding the functional constraints for sequence variability with biological implications (virulence, alteration of host range, etc.) and the population dynamics of FMDV evolution, depending on the potential structures of PKs analyzed in the present study.

An unrooted neighbor-joining tree was determined based on an alignment of the complete PK sequences (Figure 3). It was highly similar to phylogenetic trees generated from the nucleotide sequences of the VP1 and 3A regions, indicating shared evolutionary pathways under artificial conditions, and/or physical environments. Sequence analysis revealed a 47- and 42-nucleotide deletion in the PKs of OMIII (132

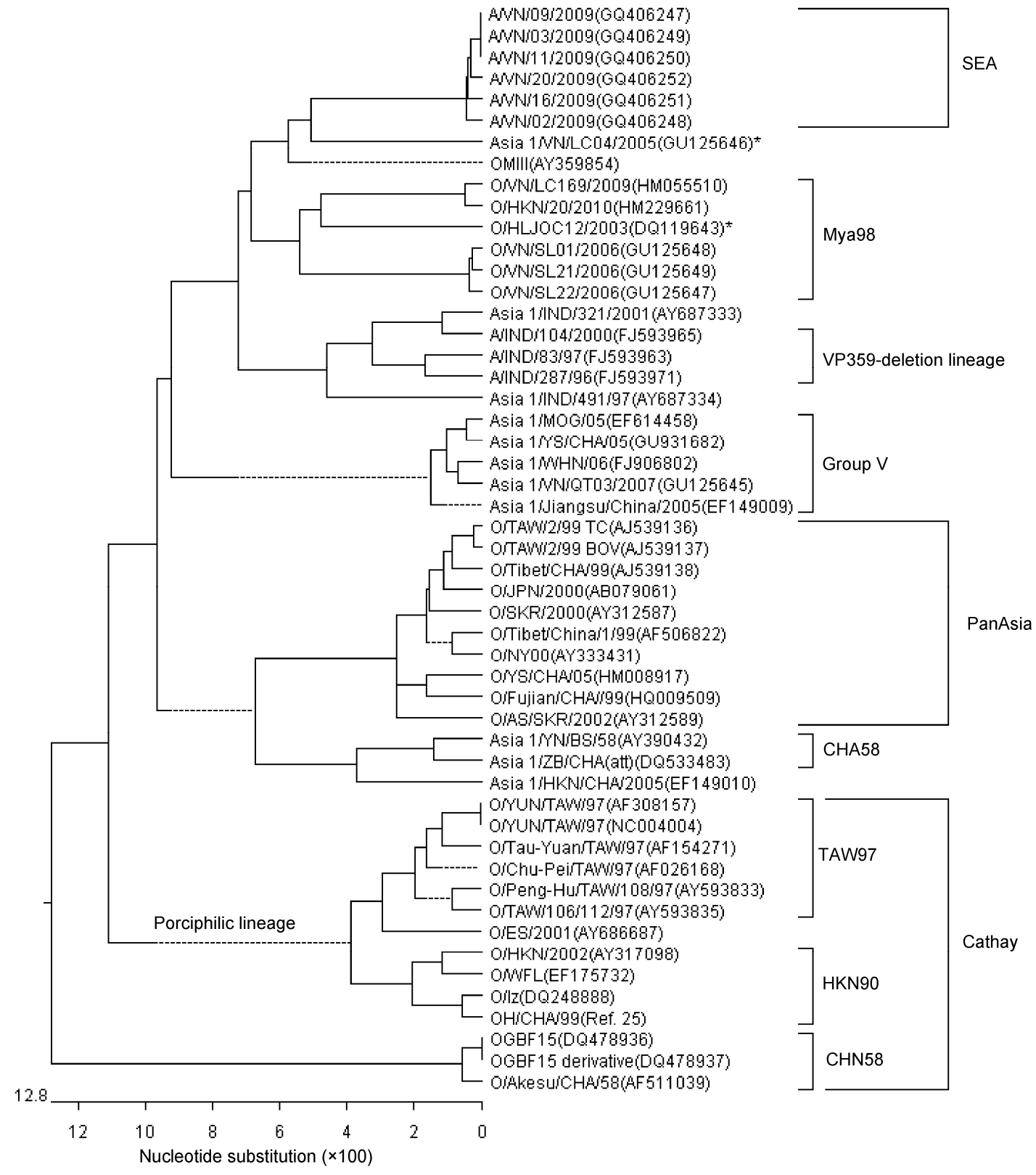

Figure 3 Unrooted neighbor-joining tree of the pseudoknot (PK) nucleotide sequences of FMDV isolates generated using the MegAlign software. * The partial PK sequence data of these isolates. 
nucleotides) and the porcinophilic lineage strains (175/176 nucleotides), respectively, compared with isolates O/Akesu/ CHA/58 (179 nucleotides) and the PanAsia strains (217/218 nucleotides). Nucleotide deletions were also detected in the PKs of serotypes A, Asia 1, C, and SAT 1-3, which may provide insight into genetic relationships between different lineages, and could be used as genetic markers [71].

The nucleotide deletions in these strains resulted in the loss of one or two of the three or four conserved PK repeats at the $5^{\prime}$-end. Remarkably, these changes may strongly hinder the process of host factor recruitment. This in turn allows the viral RNA to efficiently multiply during a relatively short infection cycle, and may affect pathogenesis and host cell tropism of FMDV. In retrospect, the "cloverleaf" structure at the non-coding region of a subgroup of picornaviruses, which include polioviruses, coxsackie B3 viruses, enteroviruses, and human rhinoviruses, implies the direct influence on the evolutionary dynamics of the 3 '-end of PKs involved in the replication of FMDV as a cis-acting element $[68,72,73]$.

\section{Recombination analysis of genetic variation, genomic mosaicism, and extension}

Complex genetic variation mechanisms are responsible for the existence of different serotypes of FMDV, resulting in altered antigenic properties and modified virulence. Phylogenetic trees generated from cross-over regions of individual isolates among and within serotypes revealed intraand/or inter-serotype recombination (A, O, C, and Asia 1) and a novel SAT lineage (which probably plays an important role in FMDV evolution and convergent evolution) will may affect both recombination detection and phylogenetic estimates [38,40,44,45,71,74-77]. Diverse breakpoint patterns occur mainly in the non-structural protein coding regions, and occasionally in the capsid coding regions.

A recent study proposed that a mosaic structure with a partial 2C fragment of Asia 1/JS/CHA/2005 was transferred from FMDV isolate Asia 1/HKN/CHA/2005 [38]. Recombination results in genetic exchange among parental viruses to generate progeny strains. This process could also explain the nonstructural protein coding region extension of FMDV genomes with degraded RdRp activity. Similarity plots and bootscanning analysis were used to assess the authentic relationships of Asia 1/HKN/CHA/05 with several reference strains. We detected the occurrence of putative gene recombination between the O/Tibet/CHA/1/99 PanAsia strain (non-coding and nonstructural protein coding regions) and the Asia 1/IND/321/01 strains (capsid coding regions). These strains recombined to produce the Asia 1/HKN/ CHA/05 strain (Figure 4). These results are analogous to the findings of previous studies, although the percentage identities of the VP1 nucleotide sequences between Asia 1/HKN/ CHA/05 and Asia 1/IND/321/01, Asia 1/IND/491/97 are $89.4 \%$ and $91.2 \%$, respectively $[13,40]$. This level of divergence with their parental genomes can be explained by the mutation rates per genome per replication cycle $[75,78,79]$. The recombination among natural FMDV populations suggests that FMDV genome diversity may exist extensively in the field [71]. Mixed infection (inter-serotype and intra-serotype) is common and positive selection occurs under natural environment, which limits the opportunity for the accumulation of genetic variation and multiple infections by different genotypes [80].

\section{Selection detection of the G-H loop in the VP1 surface capsid protein}

The capsid of FMDV presents many neutralization epitopes that cluster in several exposed regions. The G-H loop of VP1, one of the immunodominant sites, appears as a loosely connected element that may undergo hinge movements, and may adopt preferred orientations depending on the specific virus strain. The G-H loop exhibits conserved structural features. Severe restrictions on sequence variation in the $\mathrm{G}-\mathrm{H}$ loop occur because of the dual involvement of some

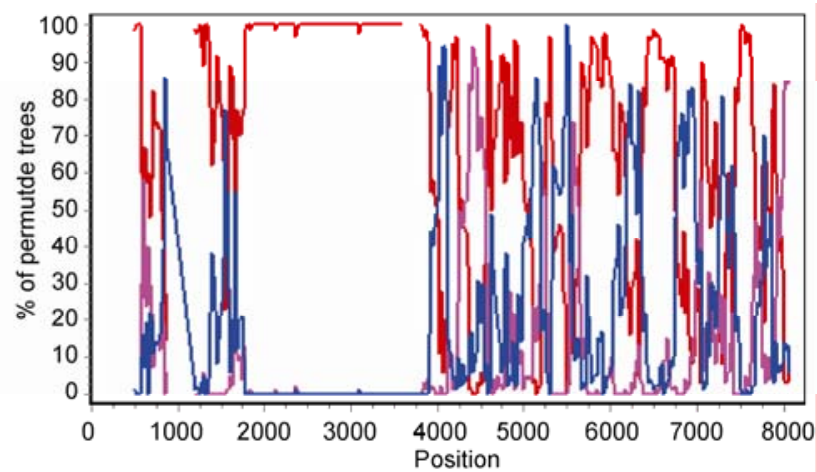

Figure 4 Similarity and bootscanning plots (http://sray.med.som.jhmi.edu/SCRoftware/SimPlot) of Asia 1/HKN/CHA/2005 in comparison with several reference strains generated using SimPlot Version 3.5.1 software [Window: 200 bp; Step: 20 bp; GapStrip: On, Kimura (2-parameter); T/t: 2.0]. 
residues, including the tripeptide RGD motif and some neighboring residues, in both antibody reactivity and cell attachment [81-84].

Phylogenetic analysis of genetic diversity and adaptive molecular evolution was also estimated under selection detection at the amino acid level for the G-H loop of serotypes $\mathrm{O}$ and Asia 1 (Table 1). In serotype $\mathrm{O}$, there were 4 residues under selection within the Cathay topotype, and seven residues under selection within the porcinophilic lineage. Residues 144 and 152 were under selection within the natural porcine-isolated PanAsia strain, and residue 135 was under selection within the natural bovine-isolated Mya98 strain. In the porcinophilic lineage, 5 residues were under stabilizing selection and four residues were under destabilizing selection within the HKN70 strain. Positive-stabilizing selection within the HKN90 and TAW97 strains occurred at 6 positions and positive-destabilizing selection occurred at 3 positions in this region. Positive selection at specific amino acid sites within the G-H loop of VP1 in serotype O isolates has been proposed to be critical in the formation of neutraliz- able antigenic sites [77,85-87]. Analysis of sequence of serotype Asia 1 strains resulted in the detection of 3 residues under stabilizing selection within Group V, 8 residues under stabilizing selection within Group II, 7 residues under stabilizing selection, and residue 140 under destabilizing selection (140Thr in Group IV and 140Pro in HKN74 strains) within the SEA topotype. Residue 136 is one of the most highly conserved amino acids in the G-H loop (with the exception of O-TW-256-2001). Residues 144, 149, and 150 are distinguishable among different serotypes, and residues 154, 155 (with the exception of O-TW-255-2000), and 158 are distinct in serotype $\mathrm{O}$ strains compared with strains of the two other serotypes. These results may contribute to our understanding of the affinity of antibody recognition and the effectiveness of synthetic peptide vaccines.

\section{Concluding remarks}

In epidemiological surveys of FMD, molecular characterization

Table 1 Selection detection of amino acid residues within the G-H loop (133-158) ${ }^{\text {a) }}$ of capsid protein VP1

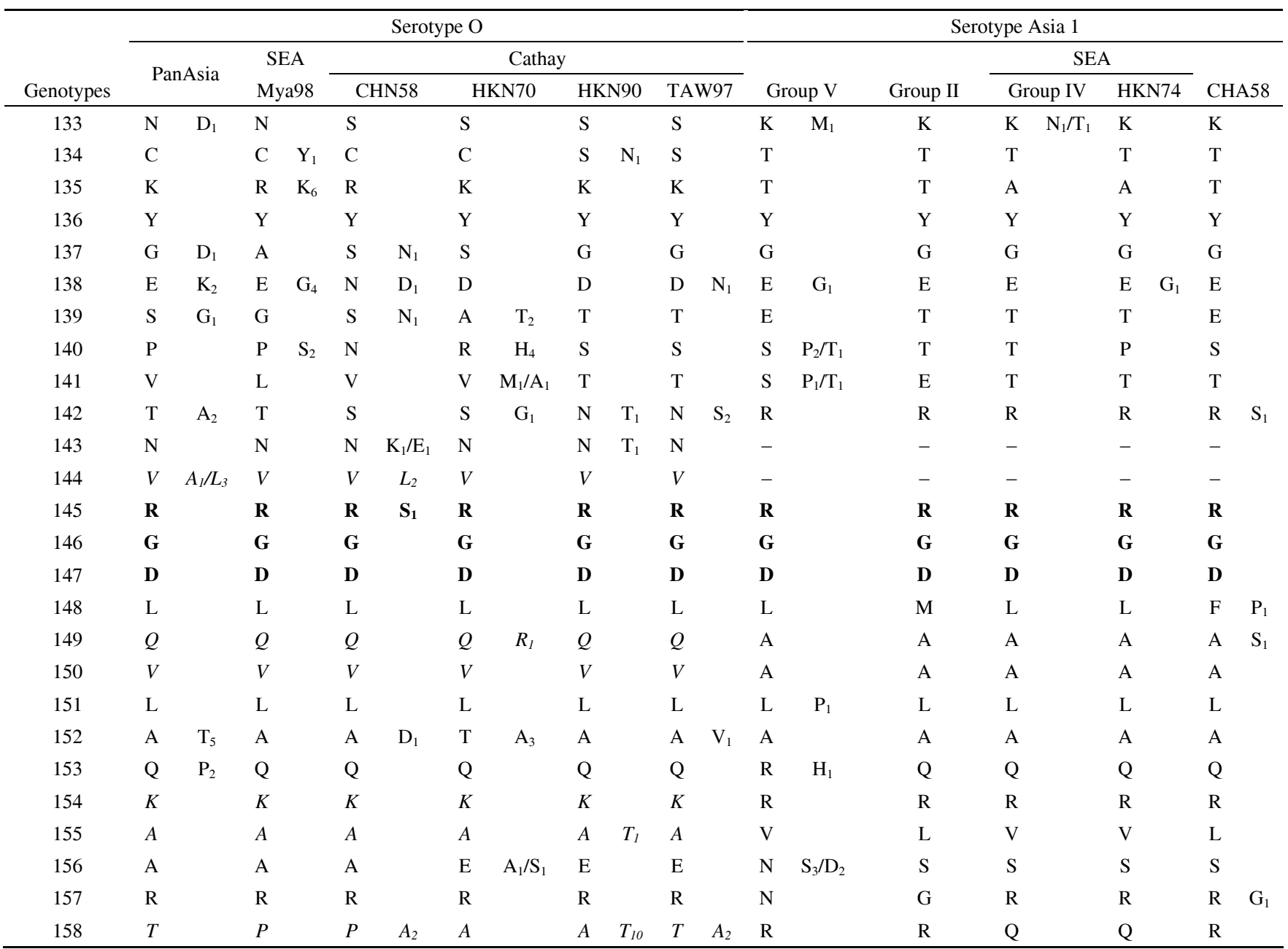

a) Residue numbers correspond to serotype $\mathrm{O}$; the most frequent amino acid for each strain is shown in the top row; specific residues for serotype $\mathrm{O}$ are shown in italics; residues of tripeptide motif within the G-H loop are shown in bold; -, residue deletion. 
of FMDV genetic diversity provided insight into the relationships between different field isolates. Despite significant advances in our understanding of viral pathogenesis and the development of new antiviral strategies in the last two decades, FMDV remains a major threat to animal husbandry in China. The control of FMD depends on: (i) strategic vaccine reserves, which can confer rapid and protective immunity in healthy and susceptible species in an outbreak (e.g., the North American Vaccine Bank and the European Union Vaccine Bank) [56]; (ii) the establishment of immune bands around national border lines to interrupt airborne transmission. FMD can be transmitted by airborne viruses many kilometers away from the virus source. It has been shown that aerosol exposure results in the typical clinical signs of FMD or persistent subclinical infections of FMDV in animals $[88,89]$. This in turn may lead to genetic drift in less fit genotypes of the virus [37]; (iii) understanding the global epidemiology of FMDV, especially in neighboring countries and areas, and implicating control measures such as transport restrictions of susceptible animals and the quarantine of animal products [90,91]; and (iv) introducing strict biosafety measures and the prudent application of innovative technologies to prevent the viruses from escaping from research laboratories [9,92]. These findings may prove beneficial in formulating control measures against FMD by the Chinese government.

We are thankful to National Foot-and-Mouth Disease Reference Laboratory of China (NFMDRLC) for providing the necessary facilities for conducting data analysis. This work was supported by National High Technology Research and Development Program of China (2011AA10A211), Ministry of Agriculture of China for Animal Disease Control in Border Areas (201003008), and the Science and Technology Program of Lanzhou, Gansu Province, China (2010-1-157).

1 Alexandersen S, Mowat N. Foot-and-mouth disease: Host range and pathogenesis. Curr Top Microbiol Immunol, 2005, 288: 9-42

2 Pereira H G. Foot-and-mouth disease. In: Gibbs E P J, ed. Virus Disease of Food Animals. London: Academic Press Inc, 1981. 333-363

3 Brown F. The history of research in foot-and-mouth disease. Virus Res, 2003, 91: 3-7

4 Domingo E, Escarmis C, Baranowski E, et al. Evolution of footand-mouth disease virus. Virus Res, 2003, 91: 47-63

5 Kitching R P. Global epidemiology and prospects for control of foot-and-mouth disease. Curr Top Microbiol Immunol, 2005, 288: $133-148$

6 Thompson D, Muriel P, Russell D, et al. Economic costs of the footand-mouth disease outbreak in the United Kingdom in 2001. Rev Sci Tech, 2002, 21: 675-687

7 Knowles N J, Samuel A R. Molecular epidemiology of foot-andmouth disease virus. Virus Res, 2003, 91: 65-80

8 Office International des Epizooties. Handistatus II. In: International Office of Epizootics, 2006

9 Cottam E M, Wadsworth J, Shaw A E, et al. Transmission pathways of foot-and-mouth disease virus in the United Kingdom in 2007. PLoS Pathog, 2008, 4: e1000050

10 Schumann K R, Knowles N J, Davies P R, et al. Genetic characterization and molecular epidemiology of foot-and-mouth disease viruses isolated form Afghanistan in 2003-2005. Virus Genes, 2008, 36:
401-413

11 Li D, Liu Z X, Bao H F, et al. The complete genome sequence of foot-and-mouth disease virus O/Akesu/58 strain and its some molecular characteristics. Arch Virol, 2007, 152: 2079-2085

12 Bai X W, Li P H, Cao Y M, et al. Engineering infectious foot-andmouth disease virus in vivo from a full-length genomic cDNA clone of the A/AKT/58 strain. Sci China C Life Sci, 2009, 52: 155-162

13 Du J Z, Chang H Y, Cong G Z, et al. Complete nucleotide sequence of a Chinese serotype Asial vaccine strain of foot-and-mouth disease virus. Virus Genes, 2007, 35: 635-642

14 Chang Y Y, Zheng H X, Shang Y J, et al. Recovery of infectious foot-and-mouth disease virus from full-length genomic cDNA clones using an RNA polymerase I system. Acta Biochim Biophys Sin, 2009, 41: 998-1007

15 Cao Y M, Lu Z J, Sun P, et al. A pseudotype baculovirus expressing the capsid protein of foot-and-mouth disease virus and a T-Cell immunogen shows enhanced immunogenicity in mice. Virol J, 2011, 8: 77

16 Valarcher J F, Knowles N J, Fernandez R, et al. FMD global update 2003-2004. In: Open Sessions of the EUFMD Research Group. Greece: Chaina, 2004

17 Knowles N J, Davies P R, Henry T, et al. Emergence in Asia of foot-and-mouth disease viruses with altered host range: Characterization of alterations in the 3A protein. J Virol, 2001, 75: 1551-1556

18 Hemadri D, Tosh C, Sanyal A, et al. Emergence of a new strain of type $\mathrm{O}$ foot-and-mouth disease virus: Its phylogenetic and evolutionary relationship with the PanAsia pandemic strain. Virus Genes, 2002, 25: 23-24

19 Mason P W, Grubman M J, Baxt B. Molecular basis of pathogenesis of FMDV. Virus Res, 2003, 91: 9-32

20 Knowles N J, Samuel A R, Davies P R, et al. Pandemic strain of foot-and-mouth disease virus serotype O. Emerg Infect Dis, 2005, 11: 1887-1893

21 Cottam E M, Haydon D T, Paton D J, et al. Molecular epidemiology of the foot-and-mouth disease virus outbreak in the United Kingdom in 2001. J Virol, 2006, 80: 11274-11282

22 Dunn C S, Donaldson A I. Natural adaption to pigs of a Taiwanese isolate of foot-and-mouth disease virus. Vet Rec, 1997, 141: 174-175

23 Beard C W, Mason P W. Genetic determinants of altered virulence of Taiwanese foot-and-mouth disease virus. J Virol, 2000, 74: 987-991

24 Grubman M J. Development of novel strategies to control foot-and-mouth disease: Marker vaccines and antivirals. Biologicals, 2005, 33: 227-234

25 Liu G Q, Liu Z X, Xie Q G, et al. Infectious foot-and-mouth disease virus derived from a cloned full-length cDNA of OH/CHA/99. Chinese Sci Bull, 2004, 49: 1137-1141

26 Liu G Q, Liu Z X, Xie Q G, et al. Generation of an infectious cDNA clone of an FMDV strain isolated from swine. Virus Res, 2004, 104: 157-164

27 Chen J D, Zhao M Q, Hui K H, et al. Molecular characterization of foot-and-mouth disease virus in Hong Kong during 2001-2002. Virus Genes, 2006, 32: 139-143

28 Zhao M Q, Suo Q L, Chen J D, et al. Sequence analysis of the protein-coding regions of foot-and-mouth disease virus $\mathrm{O} / \mathrm{HK} / 2001$. Vet Microbiol, 2008, 130: 238-246

29 Zhao Q Z, Pacheco J M, Mason P W. Evaluation of genetically engineered derivatives of a Chinese strain of foot-and-mouth disease virus reveals a novel cell-binding site which functions in cell culture and in animals. J Virol, 2003, 77: 3269-3280

30 Jin N Y, Zhang H Y, Yin G F, et al. Immunogenicity of recombinant fowl-pox virus co-expressing structural protein precursor $\mathrm{P} 1-2 \mathrm{~A}$ and proteinase 3C of FMDV. Chinese Sci Bull, 2004, 49: 823-827

31 Zhang X S, Liu Z X, Zhao Q Z, et al. Sequencing and analysis for the full-length genome RNA of foot-and-mouth disease virus China/99. Sci China C: Life Sci, 2004, 47: 74-81

32 Bai X W, Bao H F, Li P H, et al. Genetic characterization of the cell-adapted PanAsia strain of foot-and-mouth disease virus 
O/Fujian/CHA/5/99 isolated from swine. Virol J, 2010, 7: 208

33 Yang D C, Zhang C Y, Zhao L, et al. Identification of a conserved linear epitope on the VP1 protein of serotype $\mathrm{O}$ foot-and-mouth disease virus by neutralising monoclonal antibody $8 \mathrm{E} 8$. Virus Res, 2011, 155: 291-299

34 Paton D J, King D P, Knowles N J, et al. Recent spread of foot-and-mouth disease in the Far East. Vet Rec, 2010, 166: 569-570

35 Ansell D M, Samuel A R, Carpenter W C, et al. Genetic relationships between foot-and-mouth disease type Asia 1 viruses. Epidemiol Infect, 1994, 112: 213-224

36 Valarcher J F, Knowles N J, Ferris N P, et al. Recent spread of FMD virus serotype Asia 1. Vet Rec, 2005, 157: 30

37 Klein J. Understanding the molecular epidemiology of foot-andmouth-disease virus. Infect Genet Evol, 2009, 9: 153-161

38 Lee K N, Oem J K, Park J H, et al. Evidence of recombination in a new isolate of foot-and-mouth disease virus serotype Asia 1. Virus Res, 2009, 139: 117-121

39 Guo H C, Liu X T, Liu Z X, et al. Recent outbreaks of foot-andmouth disease type Asia 1 in China. J Vet Med B Infect Dis Vet Public Health, 2006, 53(suppl 1): 29-33

40 Li D, Shang Y J, Liu Z X, et al. Molecular relationships between type Asia 1 new strain from China and type $O$ Panasia strains of foot-andmouth-disease virus. Virus Genes, 2007, 35: 273-279

41 Zheng $\mathrm{H} \mathrm{X}$, Guo $\mathrm{J} \mathrm{H}$, Jin $\mathrm{Y}$, et al. Infectivity of Asia1 type foot-and-mouth disease virus was increased via an alternative RDD motif. Chinese Sci Bull, 2010, 55: 1370-1375

42 Wang $\mathrm{H} \mathrm{W}$, Zhao L, Li W, et al. Identification of a conformational epitope on the VP1 G-H Loop of type Asial foot-and-mouth disease virus defined by a protective monoclonal antibody. Vet Microbiol, 2011, 148: 189-199

43 Li D, Shang Y J, Liu Z X, et al. Comparisons of the complete genomes of two Chinese isolates of a recent foot-and-mouth disease type Asia 1 virus. Arch Virol, 2007, 152: 1699-1708

44 Tosh C, Hemadri D, Sanyal A. Evidence of recombination in the capsid-coding region of type A foot-and-mouth disease virus. J Gen Virol, 2002, 83: 2455-2460

45 Jackson A L, O'Neill H, Maree F, et al. Mosaic structure of foot-andmouth disease virus genomes. J Gen Virol, 2007, 88: 487-492

46 Ruiz-Jarabo C M, Arias A, Baranowski E, et al. Memory in viral quasispecies. J Virol, 2000, 74: 3543-3547

47 Jackson T, King A M, Stuart D I, et al. Structure and receptor binding. Virus Res, 2003, 91: 33-46

48 Verdaguer N, Mateu M G, Andreu D, et al. Structure of the major antigenic loop of foot-and-mouth disease virus complexed with a neutralizing antibody: Direct involvement of the Arg-Gly-Asp motif in the interaction. EMBO J, 1995, 14: 1690-1696

49 Domingo E, Mateu M G, Martínez M A, et al. Genetic variability and antigenic diversity of foot-and-mouth disease virus. In: Kurstak E, Marusyk R G, Murphy S A, et al. eds. Applied Virology Research, Vol 2, Virus Variation and Epidemiology. New York: Plenum Publishing Corp, 1990. 233-266

50 Pfaff M. Recognition sites of RGD-dependent integrins. In: Eble J A, Kühn K, eds. Integrin-ligand Interaction. Heidelberg: SpringerVerlag, 1997. 101-121

51 Storey P, Theron J, Maree F F, et al. A second RGD motif in the 1D capsid protein of a SAT1 type foot-and-mouth disease virus field isolate is not essential for attachment to target cells. Virus Res, 2007, 124: $184-192$

52 Tami C, Taboga O, Berinstein A, et al. Evidence of the coevolution of antigenicity and host cell tropism of foot-and-mouth disease virus in vivo. J Virol, 2003, 77: 1219-1226

53 Jackson T, Ellard F M, Ghazaleh R A, et al. Efficient infection of cells in culture by type $\mathrm{O}$ foot-and-mouth disease virus requires binding to cell surface heparin sulfate. J Virol, 1996, 70: 5282-5287

54 Mateu M G. Antibody recognition of picornaviruses and escape from neutralization: a structural review. Virus Res, 1995, 38: 1-24

55 Martínez A M, Verdaguer N, Mateu M G, et al. Evolution subverting essentiality: Dispensability of the cell attachment Arg-Gly-Asp motif in multiple passaged foot-and-mouth disease virus. Proc Natl Acad Sci USA, 1997, 94: 6798-6802

56 Sobrino F, Sáiz M, Jiménez-Clavero M A, et al. Foot-and-mouth disease virus: A long known virus, but a current threat. Vet Res, 2001, 32: $1-30$

57 Wilke C O, Novella I S. Phenotypic mixing and hiding may contribute to memory in viral quasispecies. BMC Microbiol, 2003, 3: 11

58 Saitou N, Nei M. The neighbor-joining method: A new method for reconstructing phylogenetic trees. Mol Biol Evol, 1987, 4: 406-425

59 Li J. Situation and control of FMD in mainland, China. 16th OIE Sub-Commission Meeting for FMD Control in South East Asia, 15-19 March 2010, Lao PDR: Vientiane. 2010

60 Baranowski E, Ruiz-Jarabo C M, Sevilla N, et al. Cell recognition by foot-and-mouth disease virus that lacks the RGD integrin-binding motif: Flexibility in aphthovirus receptor usage. J Virol, 2000, 74: 1641-1647

61 Giraudo A T, Beck E, Strebel K, et al. Identification of a nucleotide deletion in parts of polypeptide $3 \mathrm{~A}$ in two independent attenuated aphthovirus strains. Virology, 1990, 177: 780-783

62 Núñez J I, Baranowski E, Molina N, et al. A single amino acid substitution in nonstructural protein $3 \mathrm{~A}$ can mediate adaptation of foot-and-mouth disease virus to the guinea pig. J Virol, 2001, 75: 3977-3983

63 Baranowski E, Sevilla N, Verdeguer N, et al. Multiple virulence determinants of foot-and-mouth disease virus in cell culture. J Virol, 1998, 72: 6362-6372

64 Baranowski E, Ruiz-Jarabo C M, Domingo E. Evolution of cell recognition by viruses. Science, 2001, 292: 1102-1105

65 Oem J K, Yeh M T, McKenna T S, et al. Pathogenic characteristics of the Korean 2002 isolate of foot-and-mouth disease virus serotype O in pigs and cattle. J Comp Pathol, 2008, 138: 204-214

66 O'Donnell V K, Pacheco J M, Henry T M, et al. Subcellular distribution of the foot-and-mouth disease virus $3 \mathrm{~A}$ protein in cells infected with viruses encoding wild-type and bovine-attenuated forms of 3A. Virology, 2001, 287: 151-162

67 Clarke B E, Brown A L, Currey K M, et al. Potential secondary and tertiary structure in the genomic RNA of foot and mouth disease virus. Nucleic Acids Res, 1987, 15: 7067-7079

68 Pilipenko E V, Blinov V M, Chemov B K, et al. Conservation of the secondary structure elements of the 5 '-untranslated region of cardioand aphthovirus RNAs. Nucleic Acids Res, 1989, 17: 5701-5711

69 Escarmís C, Dopazo J, Dávila M, et al. Large deletions in the 5 -untranslated region of foot-and-mouth disease virus of serotype C. Virus Res, 1995, 35: 155-167

70 Feng Q, Yu H, Liu Y, et al. Genome comparison of a novel foot-and-mouth disease virus with other FMDV strains. Biochem Biophys Res Commun, 2004, 323: 254-263

71 Carrillo C, Tulman E R, Delhon G, et al. Comparative genomics of foot-and-mouth disease virus. J Virol, 2005, 79: 6487-6504

72 Rivera V M, Welsh J D, Jr Maizel J V. Comparative sequence analysis of the 5 ' noncoding region of the enteroviruses and rhinoviruses. Virology, 1988, 165: 42-50

73 Pilipenko E V, Maslova S V, Sinyakov A N, et al. Towards identification of cis-acting elements involved in the replication of enterovirus and rhinovirus RNAs: A proposal for the existence of tRNA-like terminal structures. Nucleic Acids Res, 1992, 20: 1739-1745

74 Krebs O, Marquardt O. Identification and characterization of footand-mouth disease virus O1 Burgwedel/1987 as an intertypic recombinant. J Gen Virol, 1992, 73: 613-619

75 Haydon D T, Bastos A D, Awadalla P. Low linkage disequilibrium indicative of recombination in foot-and-mouth disease virus gene sequence alignments. J Gen Virol, 2004, 85: 1095-1100

76 Klein J, Hussain M, Ahmad M, et al. Genetic characterisation of the recent foot-and-mouth disease virus subtype A/IRN/2005. Virol J, 2007, 4: 122

77 Lewis-Rogers N, McClellan D A, Crandall K A. The evolution of 
foot-and-mouth disease virus: Impacts of recombination and selection. Infect Genet Evol, 2008, 8: 786-798

78 Drake J W. Rates of spontaneous mutation among RNA viruses. Proc Natl Acad Sci USA, 1993, 90: 4171-4175

79 Drake J W, Holland J J. Mutation rates among RNA viruses. Proc Natl Acad Sci USA, 1999, 96: 13910-13913

80 Woodbury E L, Samuel A R, Knowles N J, et al. Analysis of mixed foot-and-mouth disease virus infections in Saudi Arabia: Prolonged circulation of an exotic serotype. Epidemiol Infect, 1994, 112: 201-211

81 Mateu M G, Martínez M A, Capucci L, et al. A single amino acid substitution affects multiple overlapping epitopes in the major antigenic site of foot-and-mouth disease virus of serotype C. J Gen Virol, 1990, 71: 629-637

82 Novella I S, Borrego B, Mateu M G, et al. Use of substituted and tandem-repeated peptides to probe the relevance of the highly conserved RGD tripeptide in the immune response against foot-andmouth disease virus. FEBS Lett, 1993, 330: 253-259

83 Verdaguer N, Sevilla N, Valero M L, et al. A similar pattern of interaction for different antibodies with a major antigenic site of foot-andmouth disease virus: Implications for intratypic antigenic variation. J Virol, 1998, 72: 739-748

84 Ruiz-Jarabo C M, Sevilla N. Dávila M, et al. Antigenic properties and population stability of a foot-and-mouth disease virus with an altered Arg-Gly-Asp receptor recognition motif. J Gen Virol, 1999, 80: 1899-1909
85 Bittle J L, Houghten R A, Alexander H, et al. Protection against foot-and-mouth disease by immunization with a chemically synthesized peptide predicted from the viral nucleotide sequence. Nature, 1982, 298: 30-33

86 Rojas E R, Carrillo E, Schiappacassi M, et al. Modification of footand-mouth disease virus $\mathrm{O} 1 \mathrm{Caseros}$ after serial passages in the presence of antiviral polyclonal sera. J Virol, 1992, 66: 3368-3372

87 Martín M J, Núñez J I, Sobrino F, et al. A procedure for detecting selection in highly variable viral genomes: Evidence of positive selection in antigenic regions of capsid protein VP1 of foot-and-mouth disease virus. J Virol Methods, 1998, 74: 215-221

88 Gloster J, Ryan E, Wright C, et al. WITHDRAWN: Foot-and-mouth disease: How much airborne virus do animals exhale? Vet J, 2008, doi: 10.1016/j.tvj1.2008.08.003

89 Pacheco J M, Arzt J, Rodriguez L L. Early events in the pathogenesis of foot-and-mouth disease in cattle after controlled aerosol exposure. Vet J, 2010, 183: 46-53

90 Nobiron I, Rémond M, Kaiser C, et al. The nucleotide sequence of foot-and-mouth disease virus O/FRA/1/2001 and comparison with its British parental strain O/UKG/35/2001. Virus Res, 2005, 108: 225229

91 Manrubia S C, Lázaro E. Viral evolution. Physics Life Rev, 2006, 3: 65-92

92 Hui E K. Reasons for the increase in emerging and re-emerging viral infectious diseases. Microbes Infect, 2006, 8: 905-916

Open Access This article is distributed under the terms of the Creative Commons Attribution License which permits any use, distribution, and reproduction in any medium, provided the original author(s) and source are credited. 